\title{
A PALEONTOLOGIA NO ESTADO DE SÃO PAULO: SUA EVOLUÇÃO E SEUS PROBLEMAS *
}

\author{
Geólogo SÉRGIO MEZZALIRA - Pesquisador Científico
}

\begin{abstract}
RESUMO
O presente trabalho é uma tentativa de efetuar um relatório histórico sobre contribuições científicas estrangeiras e nacionais à pesquisa paleontológica no Estado de São Paulo, Brasil até 1977.
\end{abstract}

\section{ABSTRACT}

This paper is an attempt to do one historical report about foreign and native scientific contributions to the paleontological researchs on the state of $\mathrm{S}$. Paulo, Brazil, up to 1977.

\section{I}

As pesquisas paleontológicas, no Estado de São Paulo, tiveram, no seu início, como praticamente em todos outros Estados do Brasil, intensa participação de pesquisadores estrangeiros.

O campo para as suas pesquisas era vasto e oferecia inúmeras oportunidades para novos descobrimentos e ampliação do conhecimento das ciências geológicas e mais particularmente a paleontologia.

Embora A. PISSIS, em 1848, mencionasse o encontro de restos organizados, muito pequenos e mal conservados, que não lhe permitiam uma definição sobre os mesmos, coube a RICHARD RATHBUN a constatação de répteis fósseis, em calcários, dos arredores de Tietê, SP, abstendo-se de opinar sobre a idade das camadas, pelo fato de o animal fóssil the ser desconhecido. Foi este cientista, incumbido pela Comissão Geográfica do Império, organizada em 1875, para realizar estudos na Província de São Paulo, tendo-a percorrido em 1877, quando teve ocasião de assinalar o encontro desses animais fósseis.

A descoberta, no calcário de Piracicaba, de poucas e mal conservadas conchas fósseis idênticas às já anteriormente observadas em Colônia Teresa, na Província do Paraná, como características dos terrenos carboníferos, confirmara a ORVILLE A. DERBY (1879) a possível semelhança de estrutura geológica das duas províncias.

A primeira real contribuição paleontológica foi a de E.D. COPE, em 1885, quando descreveu Stereosternum tumidum n. gen. n. sp. encontrado na região de Itapetininga, SP.

Os estudos geo-paleontológicos tiveram maior impulso, com a criação da Comissão Geográfica e Geológica a 27 de março de 1886, sob a direção de ORVILLE A. DERBY, tendo como colaboradores os primeiros técnicos nacionais aliados aos estrangeiros, como LUIZ F. GONZAGA DE CAMPOS, FRANCISCO DE PAULA DE OLIVEIRA, TEODORO SAMPAIO, GUILHERME FLORENCE, EUGEN HUSSAK, ALBERT LOEFGREN e mais tarde JOVIANO PACHECO, dos quais somente este último se dedicou praticamente à paleontologia. As pesquisas paleontológicas, aliadas às geológicas, se desenvolveram intensamente contribuindo para a descoberta de inúmeros jazigos fossilíferos na Província, e foram de tal monta que

* Palestra pronunciada no Instituto de Biociências, Letras e Ciências Exatas de São José do Rio Preto, SP, em novembro de 1977. 
permitiu a J. C. BRANNER escrever, em 1919, que a "geologia desse Estado havia sido estudada melhor do que a de qualquer outro Estado da União".

Após cinco anos, àquela contribuição de E. D. COPE, surgiu, em 1890, o trabalho de B. RENAULT, versando a paleoflora com a descrição específica de Lycopodiopsis derbyi, encontrado nos arredores de Piracicaba, SP.

Em 1898 A. SMITH WOODWARD traz a sua contribuição sobre os peixes fósseis de Tremembé, SP, com a descrição de Arius iheringi $\mathrm{n}$. sp.; Tetragonopterus avus, n. sp.; Tetragonopterus ligniticus n. sp.; Percichthys antiquus $n$. sp. e Acara sp.

A série de trabalhos de autores estrangeiros, prosseguiu destacando-se os de: AMEGHINO (1907) que estudou a fauna cenozóica de mamíferos das cavernas da região de Iporanga com a identificação de: Chironectes minimus (Zim.); Chrotopterus auritus Peter; Nothrotherium maquinense (Lund); Cabassus antiquus (Lund); Sclerocalyptus sp.; Acodon angustidens (Winge); Kannabateomys amblyonyx (Wagner); Proechimys fuliginosa (Wagner); Echimys spinosa (Desm.); Sylvilagus minensis Thos.; Felix aff. onssa (Linneu); Tagassus albirostris (IIl) e Mazama sp.

JORDAN, nesse mesmo ano, sinonimizou o gênero Tetragonopterus, da região de Tremembé, para Eobryconavus, que mais tarde foi transformado em Brycon avus (Woodward) por TRAVASSOS \& SANTOS (1955); J. M. MC GREGOR (1908) descreveu do Estado do Paraná, Mesosaurus brasiliensis n. sp. da Formação Irati; SOLMS-LAUBACH (1913) descreveu a madeira silicificada Tietea singularis; HERMAN VON IHERING (1913) identificou o primeiro bivalve da Formação Bauru, Pleiodon priscus; O. A. DERBY (1915) apresentou um estudo estrutural da Tietea singularis Solms-Laubach; KARL HOLDHAUS (1919) descreveu o bivalve Plesiocyprinella carinata $n$. sp. da região de Araras, SP., entre cutros do Estado do $\mathrm{Pa}$ raná; J. M. CLARKE (1920) descreveu os crustáceos Paulocaris pachecoi n. gen. n. sp. e Gampsonyx brazilicus n. sp., procedentes de Guareí, SP.; C. H. EIGENMANN \& G. S. MEYER (1929) identificaram Eobrycon branneri n. sp., de Tremembé; VON HUENE que percorreu o Estado em meados de junho de 1929, escre- veu vários trabalhos, principalmente, sobre répteis cretácicos e triássicos do Sul do Brasil, identificando em 1931 Brasileosaurus pachecoi $n$. gen. et $n$. sp. da região de Presidente Bernardes (ex-Guarucaia), SP; K. BEURLEN (1931) com material fornecido por VON HUENE, coletado em Assistência, município de Rio Claro, descreveu os crustáceos Liocaris huenei $n$. gen. et $n$. $\mathrm{sp}$. e Liocaris angusta n. sp.

Ainda, dentro dessa sequiência, temos $\mathbf{J}$. R. COWPER REED (1932) que descreveu de Rio Claro, SP., vários bivalves, Myphoriopsis brasiliensis n. sp.; Pinzonella illusa $\mathrm{n}$. gen. et $\mathrm{n}$. sp; Pinzonella similis n. sp; Ferrazia cardinalis n. gen. et $n$. sp. associados com outros já estudados do Paraná e tidos, na época, como triássicos; CARLOTA J. MAURY (1935) identificou os gastrópodes da região de Iporanga, Strophocheilus ovatus iguapensis e Thaumastus magnificus othoni; K. STAESCHE (1937) descreveu dos arredores de Araçatuba, SP., o quelônio, Podocnemis brasiliensis n. sp.; BOB SCHAEFFER (1947) reestudou os peixes actinopterígios do Brasil e identificou de Tremembé, uma nova entidade Aequidens pauloensis n. sp.; K. BEURLEN (1950) estudando a fauna Crustácea-Malacostraca de Tremembé, identificou Atyoidea tremembeensis n. sp., Palaemon sp. ident. e Parasticidae? sp.; DAVID DUNKLE \& B. SCHAEFFER (1956) identificaram o peixe Tholonotus braziliensis $n$. gen. et. $n$. sp. da região de Conchas, SP., e pertencente à Formação Corumbataí; R. KRAUSEL \& E. DOLIANITI (1958) identificaram Taxopitys alves-pintoi $\mathrm{n}$. gen. et $\mathrm{n}$. sp., de Tatuí, SP, e da flora do Irati os gêneros Polysolenoxylon tomando como holótipo Dadoxylon Whitei Maniero 1946 e Solenopitys, este último com a espécie S. paulistana $\mathrm{n}$. sp. Da região de Piracicaba, SP, descreveram Prototaxylon brasilianum $\mathrm{n}$. gen. $\mathrm{n}$. $\mathrm{sp}$. referível à Formação Corumbataí; H. S. BROOKS (1962), baseado no gênero Clarkecaris de Mezzalira (1952), propôs a família Clarkecarididae; SHIKAMA \& H. OZAKI (1966) descreveram da região de Tatuí, $\mathrm{SP}$, o réptil Brazilosaurus sanpauloensis $\mathrm{n}$. gen. et $n$. sp. da Formação Irati; W. KEGEL (1967) descreveu os rastos de bilobites do permiano, Amaralia paulistana n. gen. n. sp. encontrados em Limeira e Piracicaba, SP.; J. F. RIGBY (1968 e 1970) teceu considerações sobre as plantas encontradas em Laras, município de Laranjal 
Paulista e apresentou uma distribuição das plantas do gonduana inferior da bacia do Paraná; BRUCE RUNNEGAR \& NORMAN NEWELL (1971) revisaram a fauna permiana da Formação Estrada Nova, sinonimizando inúmeros fósseis descritos pelos autores anteriores e propugnando o ambiente marinho para a mesma.

A primeira contribuição nacional, apareceu em 1913, com a descrição por JOVIANO PACHECO, de restos de quelônios do cretáceo paulista e referíveis a Podocnemis harrisi n. sp., bem como, de outros animais encontrados associados. Dentro do critério de exposição adotado para os trabalhos alienígenas, prosseguimos com um ligeiro retrospecto sobre as contribuições dos autores indígenas.

Assim, M. G. O. ROXO, em 1929, identificou Alligator (Caiman) parahybensis n. sp. sinonimizado, em 1937, por esse autor, para Jacare parahybensis, provindo do Vale do Paraíba. Em 1936, identificou, também, restos de crocodilídeos da Formação Bauru sob a designação de Goniopholis paulistanus $n$. sp.; L. F. MORAES REGO (1936), descreveu da região de Guareí, SP., o escafópode, Dentalium florencei n. sp.; EUZEBIO P. DE OLIVEIRA, nesse mesmo ano, identificou a madeira silicificada, Dadoxylon derbyi $n$. sp. da região de Casa Branca, SP.; FERNANDO F. M. DE ALMEIDA (1944) descreveu, do Pré-Cambriano, algas, Collenia itapevensis n. sp. da região de Itapeva, SP.; em 1950, o concóstraco Acantholeaia regoi $n$. sp. da região de Rio Claro e referível à Formação Corumbataí; ainda, em 1950, publicou novo trabalho com a descrição da fauna de crustáceos encontrada no Arenito Botucatu e constante dos concóstracos: Bairdestheria barbosai $n$. sp.; Paleolimnadia petrii $n$. sp., Euestheria mendesi n. sp. e dos ostracodeo Pachecoia rodriguesi n. gen. n. sp.; Pachecoia acuminata $n$. sp. Candonopsis pyriformis n. gen. et n. sp.

Diversos artigos nacionais sobre madeira silicificada são feitos por JORDANO MANIERO, em 1944, descrevendo, Dadoxylon whitei n. sp.; em 1946, com a descrição de Dadoxylon roxoi $n$. sp. da região de Guareí, SP e, em 1951 com a de Parataxopitys brasiliana gen. et sp. n. de Rio Claro, SP., as duas primeiras referíveis à Formação Corumbataí e a última ao Irati. Ainda, em 1944, surgiu o trabalho de MENDES, com um estudo dos bivalves da Formação Co- rumbataí, da região de Rio Claro. Esse autor descreveu: Jacquesia brasiliensis (Reed) gen. n. Pseudocorbula camaquensis n. sp. Pseudocorbula triangularis $\mathrm{sp}$. $\mathrm{n}$., Pinzonella trigona n. sp.; Anodontophora intricans $\mathrm{n}$. $\mathrm{sp}$. e Pinzonellopis occidentalis (Reed) n. gen. associadas com outras entidades específicas já estudadas.

Em 1949, J. CAMARGO MENDES, descreveu novas formas permianas encontradas em testemunhos de sondagem, da região de Anhembi, Leinzia froesi n. gen. et $\mathrm{n}$. sp., e Leinzia gigantea $\mathrm{n}$. sp., para, em 1952, proceder a uma revisão da fauna da Formação Corumbataí, com descrição de novas entidades específicas, distribuídas pelas diversas zonas bioestratigráficas, a saber: Terraia aequilateralis n. sp., Roxoa intrigans $\mathrm{n}$. gen. sp., Roxoa corumbataiensis n. sp., Conesia mezzalirai n. gen. n. sp., Naiadopsis lamellosus n. gen. sp., Cowperešia anceps (Reed) n. gen., Barbosaia angulata n. gen. et n. sp., Holdhausiella elongata (Holdhaus) n. gen.; Holdhausiella almeidai n. sp., Casterella gratiosa n. gen. et $\mathrm{n}$. $\mathrm{sp}$.

Nesse mesmo ano, estudou e descreveu uma nova fauna carbonífera, da região de Capivarí, SP., constante de Crurithiris aff. C. planoconvexa (Shumard), Rhyncopora grossopunctata n. sp., Aviculopecten capivariensis $\mathrm{n}$. sp., e restos de bivalves e gastrópodes não determinados.

Em 1962, ainda esse autor, descreveu uma outra faúnula de bivalves da região de Angatuba, SP., de idade permiana e constante de Cowperesia camposi n. sp., Angatubia cowperesioides n. gen. et $n$. sp., Favalia arcuata $n$. gen. et $n$. sp., além de outros já conhecidos da Formação Corumbataí.

L.I. PRICE (1945) descreveu da região de Riolândia, SP., o crocodilídeo Baurusuchus pachecoi n. gen. et n. sp.; em 1950, de Presidente Bernardes, SP., Sphagesaurus huenei $\mathrm{n}$. gen. et n. sp.; e, em 1953, o quelônio Roxochelys wanderleyi $n$. gen. et n. sp., da região de Araçatuba, SP., todos animais pertencentes à fauna cretácica do Estado.

Dando prosseguimento a esse retrospecto, em 1950, apareceu o trabalho de F.R. MILANEZ \& DOLIANITI, com a descrição de Spiroxylon americanum n. sp., da região de Assistência, município de Rio Claro, SP. Posteriormente, essa espécie foi sinonimizada para Parataxopitys americana (Mila- 
nez \& Dolianiti). Em 1951, S. MEZZALIRA noticiou o encontro de restos de vegetais: Glossopteris sp. e Gangamopteris sp., sementes platispérmicas e pela primeira vez no Estado, a ocorrência de euripterídio Hastimima sp. da região de Capivari, SP. Esse mesmo autor em 1952, baseado na entidade específica de Gampsonyx brazilicus Clarke, criou o gênero Clarkecaris à vista de novos exemplárés de crustáceos da região de Tatuí, SP., e referíveis ao Membro Taquaral da Formação Irati. Mais tarde, em 1956, descreveu Nuculanla limai n. sp. e identificou Orbiculoidea cf. 0 . guaraunensis (E. Oliveira) encontrados em testemunhos de sondagem de Itaporanga, SP., e referíveis ao Grupo Tubarão (Carbonífero). Esse mesmo autor, em 1957, ampliou o conhecimento da fauna da Formação Corumbataí, na região de Piracicaba-LimeiraRio Claro, descrevendo Rioclaroa lefevrei n. gen. et n. sp. e Ferrazia simplicicarinata n. sp. associados aos demais bivalves já estudados e modificando, em parte, a distribuição bioestratigráfica desses animais. Ainda, S. MEZZALIRA, estudando a flora cenozóica de Vargem Grande do Sul, SP., identificou Tibouchina Izildaisabelae $\mathrm{n}$. $\mathrm{sp}$. associada a restos referíveis a Anonaceas, Rutales e Ebenales. Em 1969, noticiou o encontro, pela primeira vez no Estado, em testemunho de sondagem do Grupo Tubarão, da cidade de Boituva, SP., asas de insetos que estão sendo estudadas por Irajá Damiani Pinto. Em 1971, com o encontro de exemplares melhor conservados, de Clarkecaris, em diversas localidades do Estado, quer em afloramentos como em testemunhos de sondagem, apresentou uma descrição mais completa do mesmo, bem como outros dados sobre os demais gêneros de crustáceos da Formação Irati. Em seguida, (1974) estudou a fauna Invertebrata (moluscos e crustáceos) da Formação Bauru, com a identificação de: ? Anodontites paulistanensis n. sp.; ? Anodontites freitasi n. sp.; Monocondylaea cominatoi $\mathrm{n}$. sp.; Sancticarolis tolentinoi $\mathrm{n}$. gen. $\mathrm{n}$. sp.; ? Diplodon arrudai $\mathrm{n}$. sp.; Itaimbeia priscus (Ihering) $\mathrm{n}$. gen.; Physa aridi $\mathrm{n}$. sp.; ? Hidrobia prudentinensis n. sp., Viviparus souzai n. sp. (de Goiás) e Paleolimna diopsis suarezi $n$. sp. e sinonimiza Praechara barbosai (Petri). Nesse mesmo trabalho identificou de Minas Gerais: Anodontites pricei $\mathrm{n}$. sp. e Florenceia peiropolensis n. gen. et n. sp. Em 1976, apresentou no " $29 .^{\circ}$ Congresso Brasileiro de Geologia", nova contribuição à distribuição bioestratigráfica dos fósseis da formação Estrada Nova e assinalou, pela primeira vez, no Grupo Tubarão, no Estado, em testemunhos de sondagem da região de Sărapuí, SP., o vegetal Plumsteadiella sp.

ANA MARIA V. de CARVALHO, em 1952, identificou da mamalofáunula cenozóica de Alvares Machado, SP., restos de Lestodon trigonidens Gervais e de Toxodon $\mathrm{sp}$.

CA'RLOS DE PAULA COUTO, em 1956, descreveu o quíroptero, dos folhelhos de Tremembé, SP., Tadarida faustoi $\mathrm{n}$. $\mathrm{sp}$. e, em 1971, esse autor com S. MEZZALIRA, trouxeram notável contribuição para uma nova conceituação geocronológica dos folhelhos de Taubaté-Tremembé, com a identificação de Leontinia cf. L. gaudryi Ameghino.

SETEMBRINO PETRI, em 1955, identificou Chara barbosai n. sp. em sedimentos da Formação Bauru, da região de Machado de Melo, SP. No decorrer desse ano, H.S. TRAVASSOS \& RUBENS S. SANTOS, apresentaram uma revisão dos caracideos fósseis da bacia do Paraíba, sinonimizando as espécies conhecidas e descrevendo outras, tais como: Astyanax unicus n. sp. e Curimata mosesi n. sp.

Em 1965, ROBERTO CARDOSO, descreveu do Grupo São Bento, da região de Rifaina, SP., o concóstraco Graptoestheriella fernandoi $n$. gen. et $n$. sp.

ANTONIO ROCHA CAMPOS, em 1966 , identificou, na fauna marinha, carbonífera de Capivari, SP., o gastrópode, Peruvispira delicata Chronic; J. MARIM SUAREZ (1969) identificou o quelônio Podocnemis elegans n. sp. da região de Presidente Prudente, SP., e referível à Formação Bauru (Cretáceo); NICEIA TRINDADE (1969) assinalou a presença de megásporos na Formação Corumbataí (Permiano) com a descrição de Trileites corumbataiensis $n$. sp. DAEMON \& QUADROS estabeleceram, em 1970, uma bioestratigrafia do neopaleozóico da bacia do $\mathrm{Pa}$ ráná, baseado em esporos dispersos das turmas Saccites, Monoletes e Monocolpates, além de representantes de Tasmanales, abrangendo os Estados de Mato Grosso, Goiás, São Paulo até o Rio Grande do Sul. Do Estado, estudaram amostras dos arredores de Itu e de testemunhos de sondagem de Assistência, Paraguaçu Paulista, Guareí, Lins e Olímpia, todos poços da Petrobrás. 
F.M. ARID \& LUIZ D. VIZZOTTO (1971) identificaram o réptil Antarctosaurus brasiliensis $\mathrm{n}$. $\mathrm{sp}$. em sedimentos da Formação Bauru (Cretáceo Superior) da região de São José do Rio Preto. ANTONIO SOUZA, OSMAR SINELLI \& NEIDE MARIA M. GONÇALVES (1971) descreveram uma nova fáunula de crustáceos encontrada no Arenito Botucatu, nas proximidades de Serrana, SP. Entre os concóstracos e os ostrácodos estudados e determinados, foram propostas duas novads espécies: Euestheria ribeiropretensis n. sp. e Euestheria triangularis $\mathrm{n}$. $\mathrm{sp}$. associadas a Lioestheria elliptica (Jones), Pseudestheria sp., Estheriella sp., Cypridea oblonga (?) e ao vegetal Lycopodiopsis derbyi Renault. SERGIO E. AMARAL assinalbu (1971) a ocorrência de espículas de esponjas, Demospongea, ordem Haplosclerida ou Poecilosclerida, em toda a extensão vertical do Passa Dois, bem como, esporos de plantas.

Em 1973, PAULA COUTO reestudou a fauna cenozóica relativa aos edentados fóst seis das regiões de Ālvares Machado, SP., Sorocaba-Iporanga e das cavernas do Vale do rio Ribeira, sinonimizando e redescrevendo algumas formas. EVALDO RAGONHA \& PAULO SOARES assinalaram (1974) a ocorrência de gyrogonites de algas Charačeas na Formação Estrada Nova, em Anhembi, SP., que lembram Leonardosia langei Sommer do Paraná. Ainda, nesse mesmo ano, CÂNDIDO FERREIRA identificou nas argilas bentoníticas de Tremembé, SP., gastropódos pulmonados d'água doce, Lymmea sp. e Biomphalaria, referindo esta a B. cf. B. glabrata (Say 1818).

DIANA MUSSA descreveu (1974) novo gênero de madeira fóssil da Formação Irati, da região de Piracicaba, denominando-o de Protopodocarpytis rossleri $n$. gen. et $n$. sp. IGNÂCIO BRITO \& MAURO RIBEIRO identificaram (1975) a ocorrência de Lepidóptero - Danaidae Doubleday 1847 nos folhelhos de Tremembé, achando muita semelhança das nervuras do fossíl com as do gênero Anosia. J. MILLAN (1975) publicou seu trabalho sobre a tafoflórula de Monte Mor, SP. assinalando pela primeira vez, no Grupo Tubarão do Estado e no Gonduana inferior do Brasil inúmeros exemplares de vegetais fósseis, já descritos e contribui com a descrição de Paracalamites montemorensis $\mathrm{sp}$. n. e Cordaicarpus nitens (Feruglio) comb. nova.
DINA ARÁUJO, do Rio Grande do Sul apresentou, em 1976, um estudo estatístico e osteológico dos Progranosáuria da bacia do Paraná, concluindo pela validade dos três gêneros: Mesosaurus, Stereosternum e Brazilosaurus.

No "29.0 Congresso Brasileiro de Geologia" (1976) há vários trabalhos que versam a paleontologia do Estado, destacando-se os de MURILO LIMA et alii sobre os foraminíferos arenáceos do Subgrupo Itararé; DIANA MUSSA sobre as Vertebraria da formação Irati; GIUSEPPE LEONARDI sobre a descoberta de uma rica icnofauna (Vertebrados e Invertebrados) no Arenito Botucatu em Araraquara, e o estudo de PAULA COUTO sobre a paleontologia e cronogeologia da bacia Tremembé-Taubaté.

No "I Simpósio Regional de Geologia" (1977) patrocinado pelo Núcleo de São Paulo, da Sociedade Brasileira de Geologia, dentre os trabalhos apresentados destacamos: o de K. SUGUIO \& DIANA MUSSA que descreveram as seguintes madeiras fósseis dos aluviões antigos do rio Tietê: Astronioxylon mainieri; Piptadeni oxylon chimeloi; Mirocarpoxylon sanpaulensis; Matayboxylon tietei e Qualeoxylon itaquaquecetubai, todas referíveis aos Pleistoceno Sup.; o de GIUSEPPE LEONARDI sobre a ocorrência de Titanosauridae na Formação Bauru em Guararapes, SP., e o de FAIRCHILD que tratou dos Conophyton and other columnar stromatolites from Proterozoic Açungui Group near Itapeva, SP., Brazil.

Nesse ligeiro retrospecto demos ênfase às contribuições, indígenas e alienígenas, que trouxeram a lume, novas entidades genéricas e/ou específicas, não desmerecendo as demais que, também, têm o seu valor para a evolução das pesquisas paleontológicas, ampliando a distribuição geográfica dos fósseis por todo o Estado, mas que se fossem aqui enunciadas tornaria esse retrospecto por demais longo.

\section{II}

As únicas Instituições que poderiam incrementar a formação dos Geólogos, especialistas em paleontologia, em face da legislação vigente, com aproveitamento nos serviços públicos, seriam os Institutos de Geociências e Biociências. Infelizmente, isso parece não estar acontecendo e os pou- 
cos Geólogos, taivez, $2 \%$ dos diplomados nesses últimos 10 anos, que se iniciaram em paleontologia, bandearam-se para outras disciplinas, tais como: Geologia Econômica, Geologia Aplicada, Hidrogeologia, Geofísica, etc., onde a possibilidade de melhor remuneração é bem maior.

Quais seriam as causas desse desinteresse que temos notado, principalmente em alunos estagiários e a desistência que se vem observando naqueles que se iniciaram no campo da paleontologia? A nosso ver, essas causas seriam:

1. Problemas relativos ao ensino da Paleontologia;

2. Ausência de mercado de trabalho;

3. Baixa remuneração desestimulando o principiante, 'se bem que esse problema seja de caráter geral.

No que diz respeito ao primeiro item, nada podemos opinar, pois como não estamos ligado diretamente à docência, desconhecemos a/ou as causas ou deficiência observadas no ensino da paleontologia.

O que desejamos salientar é que alunos, de diversas Universidades, que têm estagiado no Instituto Geológico, demonstraram um total desinteresse pela paleontologia, não desejando mesmo nenhum trabalho sobre o assunto. Sabemos que o Geólogo não é natưralista e o naturalista nem sempre é Geólogo, daí, talvez, a causa do problema em tela, sendo por isso, necessário uma revisão no processo de ensino.

A ausência de mercado para o especia.lista em paleontologia é bastante sentida e ele só poderá exercer sua profissão, em entidade pública e/ou autárquica, contendo em sua organização, seções especializadas, ou então nas Universidades, no ensino. No âmbito particular o paleontólogo não tem chance nenhuma. O que se observa, atualmente, pelo menos no Estado de São Paulo, é a inexistência de renovação de valores; os trabalhos paleontológicos, via de regra, são sempre dos mesmos autores, já tradicionais e, quando aparecem outros, de novos especialistas, verifica-se com o correr dos anos, a mudança radical para as ciências afins, tornando-se a paleontologia um passatempo e, isso, é ocasionado por circunstâncias diversas, dentre as quais a própria subsistência.

A remunèração, que parece ser de caráter 'geral, afetando todas as profissões liberais, principalmente nos serviços públicos, é menor e nem sempre condiz com a do mercado particular. A baixa remuneração, no início da carreira, associada à falta de mercado, provocam desestímulo na formação de paleontólogos e a desistência dos possíveis iniciantes, que vêem nas ciências afins, a possibilidade de obter melhor remuneração.

Recentemente, houve por bem o Governo do Estado, amparar, com melhor remuneração, todo o pessoal de nível superior dos Institutos de Pesquisas, com a criação da Carreira de Pesquisador Científico (Lei Complementar n. ${ }^{\circ} 125$, de 18.11 .1975 ).

E preciso, porém, dar maior divulgação do que seja a paleontologia, chamando a atenção para o papel que os fósseis apresentam na solução de problemas de cunho biológico, geológico, litoestratigráfico, paleoecológico e econômico (petróleo, carvão, etc.). 